\title{
El partido-movimiento como expresión de la democracia radical. Emergencia y consolidación de Podemos en España y Revolución Democrática en Chile
}

\author{
Eduardo Alvarado-Espina ${ }^{1}$, Rommy Morales-Olivares ${ }^{2}$ y Pablo Rivera-Vargas ${ }^{3}$
}

Recibido: 04-07-2018/ Aceptado: 22-11-2019

Resumen. La crisis económica del 2008, cuyos peores efectos se percibieron en los países desarrollados, reavivó la disputa política por la hegemonía ideológica. Con ella se evidenció la disfunción existente entre bienes materiales e inmateriales que produce la sociedad capitalista y la satisfacción de la ciudadanía hacia estos en las democracias consolidadas. Esta disfunción, como sucedió en España y Chile, originó nuevos movimientos políticos que buscaron representar las viejas injusticias irresueltas por la democracia liberal, y las nuevas tensiones que emergieron con y tras la crisis económica. El presente artículo aborda este proceso mediante el análisis comparado de una conjunción de factores estructurales y supraestructurales, con el fin de comprender la emergencia y consolidación de Podemos en España y Revolución Democrática en Chile como partidos-movimiento. Para conseguir este objetivo, y desde un marco teórico centrado en las premisas de la democracia radical y el pluralismo agonista y las características del partido-movimiento, se explora en las condiciones socioeconómicas, culturales e institucionales que pueden explicar la irrupción y consolidación de este tipo de partido político. A partir de este análisis se concluye que: 1) la emergencia de ambos partidos se explica por las condiciones materiales de desigualdad en ambos países; 2) en su origen, los dos partidos pueden ser definidos nítidamente como partidos-movimiento; y 3 ) estos partidos se han convertido en una alternativa a los partidos tradicionales, pero dentro del propio régimen político que impugnan.

Palabras clave: partidos; democracia; desigualdad; pluralismo agonista; cultura política.

\section{[en] The Movement-Party with the Expression of Radical Democracy. The Cases of Emergence and Consolidation of Podemos in Spain and Revolución Democrática in Chile}

\footnotetext{
1 Universidad de Chile (Chile).

E-mail: ealvarado@dii.uchile.cl

2 Universidad de Barcelona (España) y Wits University (Sudáfrica).

E-mail: rommymorales@ub.edu

3 Universidad de Barcelona (España) y Universidad Andrés Bello (Chile).

E-mail: pablorivera@ub.edu
}

Abstract. The financial crisis of 2008, whose worst effects were felt in developed countries, reignited the political dispute over ideological hegemony. It showed the dysfunction existing between material and immaterial goods produced by capitalist society, and the citizenry's satisfaction with these goods in consolidated democracies. In Spain and Chile, this dysfunction originated new political movements that sought to represent the old unresolved injustices for liberal democracy, and the new 
tensions that emerged with and after the economic crisis. This article addresses this process through the comparative analysis of a conjunction of structural and supra-structural factors, in order to understand the emergence and consolidation of Podemos in Spain and the Democratic Revolution in Chile as movement-parties. To achieve this goal, and based on a theoretical framework focused on the premises of radical democracy and agonist pluralism and the characteristics of the party-movement, we explore the socioeconomic, cultural and institutional conditions that may explain the emergence and consolidation of this type of "political parties." Based on this analysis, it is concluded that: 1) the emergence of both parties is explained by the material conditions of inequality in both countries; 2) in their origins, the two parties can be clearly defined as movement-parties; and 3) these parties have become an alternative to traditional parties, but within the political regime they are challenging.

Keywords: parties; democracy; inequality; agonist pluralism; political culture.

Cómo citar: Alvarado-Espina, E., R. Morales-Olivares y P. Rivera-Vargas (2020): “El partido-movimiento como expresión de la democracia radical. Emergencia y consolidación de Podemos en España y Revolución Democrática en Chile", Política y Sociedad, 57(1), pp. 21-43.

Sumario. 1. Introducción. 2. Democracia radical, pluralismo agonista y el partido-movimiento. 3. Factores o condiciones estructurales que explican la emergencia de nuevos partidos políticos. 4. Factores institucionales y no institucionales de consolidación. 5. Conclusiones. 6. Bibliografía.

\section{Introducción}

Los movimientos de protesta social que surgieron en el año 2011 en varias democracias tuvieron como denominador común una narrativa de resistencia e impugnación ciudadana a un modelo económico que ha traído desigualdad e incertidumbre a amplios sectores de la sociedad; algo que se hizo más patente con la Gran Recesión del 2008. Sin embargo, tras el entusiasmo inicial que estos movimientos despertaron, su derrotero y consolidación política no acabó siendo simétrica en todos los casos.

En la etapa postconflicto comenzó a visibilizarse la distancia entre las identidades internas y particularidades culturales de cada uno de estos movimientos, sobre todo a la hora de intentar constituirse como organizaciones políticas. En algunos casos estos conflictos se disolvieron, otros se disiparon, otros fortalecieron alternativas políticas preexistentes y, finalmente, algunos inspiraron la creación de nuevos partidos políticos. La emergencia de nuevos actores políticos es propicia cuando predomina un escenario de soterrada crisis de la estructura social y política, que otorga fuerza de empuje a las tensiones que se mantenían latentes en la interacción entre lo social y lo político. A partir de esta hipótesis, junto a los elementos que definen la particular realidad política de cada país, en el presente trabajo se analizan dos organizaciones políticas que se constituyen en España y Chile en el último lustro: Podemos y Revolución Democrática, respectivamente.

El análisis comparado que se propone se construye a partir de tres interrogantes que resultan claves para explicar la emergencia y consolidación de estos dos partidos, cuyo origen primigenio se encuentra en el contexto de movilización social del año 2011. En primer lugar, ¿cuáles fueron los factores estructurales determinantes para que los movimientos sociales dieran paso a la formación de nuevos partidos políticos? En segundo lugar, ¿se puede definir a Podemos y Revolución Democrática como partidos-movimiento? Y en tercer lugar, ¿estos nuevos actores políticos se han consolidado como una alternativa a los partidos tradicionales? 
Para responder a estas cuestiones se ha llevado a cabo un análisis de datos secundarios para el periodo 2008-2017, utilizando las siguientes fuentes de información: el índice Gini de desigualdad publicado tanto por la OCDE como por el Banco Mundial, los estudios anuales de opinión pública de Latinobarómetro y el CIS, las acciones políticas no convencionales del estudio de valores de World Values Survey y los resultados electorales del periodo 2011-2017. Todas estas fuentes dan cuenta de la estructura social y comportamiento político en ambos países. Para interpretar estos datos, se recurre a un marco teórico que combina el enfoque de la democracia radical y agonista (Laclau y Mouffe, 1987; Mouffe, 1999; 2012; Laclau, 2005) con la noción de partido-movimiento de Kitschelt (2006). De este tratamiento teórico emergieron cuatro factores relevantes para el análisis, estos son: 1) condiciones socioeconómicas que pueden impedir a un sector importante de la ciudadanía estar representada en las instituciones; 2) ruptura político-cultural entre las nuevas demandas de los electores y la disposición de los partidos tradicionales a representarlas; 3) bajos umbrales institucionales, ya sean formales o informales, que pueden favorecer la consolidación del partido-movimiento; y 4) acciones políticas disruptivas y no convencionales que desafían el relato hegemónico predominante. Por lo demás, factores que responden a dos grandes dimensiones de la organización social, la cultura política y la política económica.

El presente artículo también pretende ser un aporte teórico-empírico al estudio comparado del cambio político en sociedades heterogéneas. Sin la aparición de nuevos actores políticos con un discurso que antagonizó con el consenso político vigente, no es posible entender este cambio. Por tal motivo, el análisis gira en torno a la comparación de Podemos, cuyo origen, modelo e identidad política ha sido materia de estudio en otros trabajos previos (véanse Martín, 2015; Franzé 2017; Meyenberg, 2017); y Revolución Democrática, una formación política chilena escasamente analizada en estos aspectos.

El artículo se organiza en cuatro apartados. En el primero se desarrolla el enfoque y definen los conceptos y factores que se utilizan para el análisis comparado. En el segundo, se analizan las condiciones estructurales que inciden en la emergencia de nuevas fuerzas políticas de aparente carácter contrahegemónico, tales como la desigualdad socioeconómica y la pérdida de valor social del consenso político-cultural proveniente de las respectivas transiciones a la democracia. En el tercero, se analizan los factores institucionales (sufragio activo y pasivo) y no institucionales (acciones políticas disruptivas) que pueden posibilitar la consolidación de Podemos y Revolución Democrática. Finalmente, en el último apartado se responde a los interrogantes planteados, además de esbozar algunas conclusiones al respecto.

\section{Democracia radical, pluralismo agonista y el partido-movimiento}

El enfoque de la democracia radical básicamente postula que la democracia se constituye desde la tensión entre lo político y la política. Una tensión conducente a que la relación entre los sujetos que deciden y aquellos afectados por la decisión no sea pacífica sino más bien conflictiva. Dicho de otro modo, la relación entre representados y representantes se entiende compleja, asimétrica y antagónica. $\mathrm{Su}$ 
dialéctica estriba en que "el problema de lo político es el problema de la institución de lo social, es decir, de la definición y articulación de las relaciones sociales en un campo surcado por antagonismos" (Laclau y Mouffe, 1987: 195). A partir de lo político, se define la relación amigo-enemigo, que hace incompatible las demandas contrahegemónicas con el orden que estas cuestionan (Franzé, 2017). Una relación que en la concepción agonista pasa a definirse como un enfrentamiento entre adversarios (Mouffe, 1999; 2012). En ella la adversarialidad de lo político no se traduce en la incompatibilidad absoluta entre hegemonía y contrahegemonía, sino en una mutua influencia en la lucha democrática.

En términos teóricos, una estrategia política que combina la tradición radical y agonista de la democracia cuestiona la concepción liberal de la democracia y la política basada en el consenso racional construido en torno a las instituciones políticas. Un tipo de consenso que fija lo que está dentro y lo que está fuera del régimen democrático (Mouffe, 2012). Al fin y al cabo, la política es la lucha por el sentido que constituye la comunidad, el orden político y sus actores, no una creación externa y atemporal que limita esa lucha (Laclau y Mouffe, 1987; Franzé, 2017):

Desde esta perspectiva, el centro de gravedad de la democracia se traslada a las pasiones, valores, identidades e intereses diferenciadores - antagonismos- que dan vida a lo social y a lo político (Mouffe, 1999; 2012). En otras palabras, se perfila la universalidad de la particularidad como expresión de la política, donde la colectivización de las causas individuales o subjetivas se consigue mediante un nuevo sentido de época o nueva hegemonía. A pesar de los consensos de las supuestas mayorías, esa nueva hegemonía se sedimenta en tanto se mantenga abierta la posibilidad vinculante de impugnar las funciones o contenidos del régimen político (Laclau, 2005).

A partir de las premisas de este marco de referencia, se puede decir que en la reapertura del debate de lo político en España y Chile en 2011, subyace una crítica a los límites institucionales fijados por sus transiciones a la democracia, que dan lugar a una reinterpretación de las formas políticas imperantes. Las instituciones sociales postransicionales son un conjunto de reglas y recursos relativamente permanentes que las personas pueden tomar como punto de referencia de las acciones, en tanto impliquen continuidad o cuestionamiento (Leroux y Morales, 2016) durante la etapa de construcción política postautoritaria.

La democracia radical plantea subvertir estas reglas como posibilidad de transformación, mediante la consolidación de espacios que permitan abrirse a la interpretación y a la diferencia, potenciando el desplazamiento de las hegemonías. En ella, la idea de poder queda expuesta a la indeterminación del juego político (Mouffe, 1998). La movilidad de contenidos que produce la lucha por la hegemonía permite observar una afinidad electiva entre democracia radical-agonista y emergencia de movimientos políticos plurales que pueden constituirse en partidos-movimiento. En el caso de España y Chile, sucede que los nuevos partidos traducen la impugnación al discurso hegemónico de las élites convencionales —empresarios, políticos y medios de comunicación-, llevada a cabo por los movimientos sociales en 2011, en un programa de confrontación política que da continuidad institucional a dicha impugnación. Esto implicó dar representación política a amplias y diversas demandas sociales que no estaban en la agenda de las élites políticas tradicionales (derecho a la vivienda, derecho a una 
educación gratuita y de calidad, asamblea constituyente, regulación del sistema financiero, igualdad de género, protección del medio ambiente, corrupción).

En términos descriptivos, un partido-movimiento puede condensar diversas demandas en un programa político amplio, siguiendo un estilo organizacional preferentemente basado en un proceso de toma de decisiones participativo, flexible y horizontal, propio de los movimientos sociales, lo que les diferencia de los partidos tradicionales, más rígidos y verticales (Kitschelt, 2006; Martín, 2015). Esto le otorga un carácter mixto y de tránsito desde un movimiento social a un partido. Por tanto, el partido-movimiento es la cristalización de la transición de una etapa a otra (Kitschelt, 2006). Otra de sus características es que su acción política se corresponde a un proceso no lineal de articulación, donde las dimensiones del proyecto son las que determinan su estrategia.

$\mathrm{Su}$ conformación se produce por la asociación de los activistas políticos provenientes de movimientos sociales, quienes intentan aplicar las prácticas organizativas y estratégicas de estos movimientos en el partido. Esto implica que: 1) la inversión en la estructura organizativa formal del partido es secundaria a los aspectos programáticos; y 2) las diferentes partes del movimiento pueden estar dirigidas por un líder carismático, y al mismo tiempo coexistir con una base democrática y un sistema de coordinación participativa entre activistas (Kitschelt, 2006).

Estas características del partido-movimiento conducen a un programa de preferencias colectivas incompletas, lo que encaja con uno de los elementos normativos centrales de la democracia radical, la apertura al otro radical; el escenario democrático donde se expresa la iterabilidad y se construye un presente político transformado - a cada instante- desde el activismo, en su estructura y en su contenido. Se manifiesta la actualidad de la democracia y su indeterminación del poder, que es la que permite una democracia en permanente construcción y abierta a la alteridad posible.

Además, al conjugar los postulados de la democracia radical con la noción de partido-movimiento, se valida la tesis de Katz y Mair (2004), en cuanto a que existe un proceso dialéctico donde cada nuevo tipo de partido genera una reacción que estimula un nuevo desarrollo, y que deriva a su vez en un nuevo tipo de partido. En este caso, el nuevo tipo de partido entraría a discutir la supremacía del modelo vigente, el partido cartel. Un modelo de partido que se distingue por unos fines políticos autorreferenciales y una competencia política limitada (Katz y Mair, 2004).

Atendiendo a estas pautas, la probabilidad de que emerja una forma de partido-movimiento aumenta cuando se cumplen ciertas condiciones, a saber: 1) los intereses de un amplio sector de electores, que están dispuestos a articular sus demandas a través de actividades disruptivas y no convencionales, no se sienten representados en las instituciones; 2) los partidos tradicionales no están dispuestos a asumir tales intereses por temor a dividir su propia base electoral; y 3) las barreras - umbrales - formales e informales de acceso a la competencia electoral van de moderadas a bajas (Kitschelt, 2006: 283).

El enfoque de la democracia radical adjudica un rol sustantivo al pluralismo en la relación de lo político y la política. En otros términos, son los antagonismos de lo social y las instituciones procedimentales en disputa los que permiten la democracia radical. Es en este escenario donde se pueden visibilizar las condiciones que facilitan la emergencia de los partidos-movimiento. A partir de 
esto, se plantea un análisis sustentando en cuatro factores que permiten comparar y comprender la emergencia y consolidación de Podemos en España y de Revolución Democrática en Chile, como ejemplos empíricos de esta configuración política.

El primer factor hace referencia a las condiciones socioeconómicas que afectan lo político, y cómo desde ahí se estructura el conflicto de poder en las sociedades plurales (Mouffe, 2012). Son unas condiciones que pueden dificultar que ciertos valores e intereses que cuentan con un amplio apoyo ciudadano estén representados en las instituciones políticas. El segundo factor tiene que ver con cierto agotamiento del consentimiento y confianza de la ciudadanía en la dinámica y funcionamiento de las instituciones políticas, incluyendo a sus élites; esto es, el quiebre en la relación cultural entre estructura y superestructura, entre ideología y sentido común (Gramsci, 1975; Portelli, 1977). A esta ruptura contribuye la divergencia entre las demandas mayoritarias de la ciudadanía y las prioridades políticas de los partidos tradicionales. Se intuye que ambos factores, uno estructural y otro ideológico, facilitaron el surgimiento de nuevos partidos al margen de los parámetros formales del sistema político.

Los otros dos factores buscan explicar la posibilidad de consolidar proyectos políticos que fluyen de abajo hacia arriba y desde lo extrainstitucional a lo institucional. De este modo, un tercer factor atiende a la génesis e instalación de los nuevos partidos políticos. Esto corresponde a la forma desde la cual emana y legitima el nuevo partido, como movimientos sociales, sindicatos, organizaciones sectoriales, incluyendo la cosificación de su organización y sintonía con la ciudadanía. Aquí se tornan relevantes las acciones políticas disruptivas y no convencionales propias de los movimientos sociales. El cuarto factor tiene que ver con el encaje de partidos políticos que asimilaron el estilo y proyecto de los movimientos sociales, y que se autoperciben contrahegemónicos. Para ello, es importante conocer las posibilidades que ofrecía el sistema político para su consolidación, si las barreras formales e informales que existen para la competencia electoral no son un problema para que la primigenia legitimidad social de los nuevos actores políticos se convierta en representación política.

\section{Factores o condiciones estructurales que explican la emergencia de nuevos partidos políticos}

\subsection{Condiciones socioeconómicas desiguales que impiden la representación institucional del pluralismo}

Las condiciones socioeconómicas en España y Chile, cuando irrumpen con fuerza el $15 \mathrm{M}$ y el movimiento estudiantil del 2011, son divergentes. Según datos de la OCDE (2016), en España la desigualdad social se incrementó tras la crisis económica de 2008, mientras que en Chile esta se redujo debido principalmente a la bonanza que generó el precio del cobre y las políticas anticíclicas del primer Gobierno de Michelle Bachelet (2006-2010). Sin embargo, las diferencias estructurales siguen siendo ostensibles, como lo refleja el índice de desigualdad Gini de 0,53 en Chile y 0,34 en España ${ }^{4}$. Asimismo, y a pesar de la implantación de medidas económicas

$4 \quad$ Este es el promedio observado en los dos países para el periodo 2008-2014. 
regresivas en la distribución del ingreso (aumento del IVA y recortes sociales) por parte del Gobierno del Partido Popular (derecha política), el nivel de bienestar de España siguió siendo superior al de Chile. Aun así, cabe destacar que en ambos países los niveles de desigualdad son superiores al promedio de los países miembros de la $\mathrm{OCDE}^{5}$. Un segundo elemento a considerar es el gasto social en educación, salud y pensiones. De acuerdo a Piketty (2015), este tipo de gastos favorece la transferencia de ingresos y la redistribución de ricos a pobres. Su importancia radica en que unas malas condiciones de vida pueden afectar negativamente la valoración de la democracia y su calidad (Merkel, 2014; Alvarado-Espina, 2017).

En la Tabla 1 se ve el gasto realizado en cada una de estas áreas en el periodo 2008-2011.

Tabla 1. Porcentaje de gasto social en \% del PIB en España y Chile: 2008-2011

\begin{tabular}{|l|l|c|c|c|c|c|}
\hline \multirow{2}{*}{ País } & Tipo de gasto & 2008 & 2009 & 2010 & 2011 & Media \\
\hline \multirow{3}{*}{ Chile } & Educación & 3,8 & 4,2 & 4,2 & 4,1 & 4,1 \\
\cline { 2 - 7 } & Salud & 6,9 & 7,4 & 7 & 7 & 7,1 \\
\cline { 2 - 7 } & Pensiones & 3,3 & 3,5 & 3,4 & 3,2 & 3,4 \\
\hline \multirow{3}{*}{ España } & Educación & 4,5 & 4,9 & 4,8 & 4,8 & 4,8 \\
\cline { 2 - 7 } & Salud & 8,8 & 9,5 & 9,6 & 9,5 & 9,4 \\
\cline { 2 - 7 } & Pensiones & 8,6 & 9,5 & 10 & 11 & 9,7 \\
\hline
\end{tabular}

Fuente: elaboración propia a partir de los datos obtenidos en: https://data.oecd.org/socialexp/social-spending.htmy

La alta desigualdad socioeconómica es un factor que incide en el malestar o disconformidad del ciudadano medio hacia el sistema político (Boix, 1996). Esto se produce debido a que los individuos perciben que el sistema político no es capaz de resolver sus problemas materiales. Si a esto se añade un bajo gasto público en áreas básicas del bienestar, se constituye un marco justificatorio para toda movilización social que desafíe el statu quo, especialmente en sociedades con un fuerte componente organizador desde el Estado, como lo son Chile y España. Por tanto, la combinación de ambos hechos puede crear un escenario propicio para reivindicar los antagonismos, desde lo social a lo político, como sugiere la estrategia de la democracia radical.

En la praxis, si el malestar social que provoca una precarización de la vida en los estratos socioeconómicos medios y bajos no es asumido por los actores políticos institucionales - Gobierno, parlamento, partidos-, acaba canalizándose mediante acciones sociales y políticas no convencionales que antagonizan con el poder otorgado y la legitimidad de las élites gobernantes. 
La prolongada desigualdad en Chile - escasamente mitigada por los distintos Gobiernos - y su significativo avance en España en los últimos años brindó significado y legitimidad a una acción colectiva que consiguió ensanchar el marco del antagonismo político. La escasa sensibilidad y atención de las élites políticas tradicionales a quienes fueron más castigados por la crisis económica, o las afecciones de un sector mayoritario de la población, permitió la emergencia de movimientos políticos que impugnaron —e impugnan - la hegemonía de la ideología dominante, siguiendo las reglas del juego democrático.

En España la convergencia de individuos provenientes de distintos sectores sociales, etarios, ideológicos y profesionales se cristalizó en acciones de protesta generalizadas, cuya explicación está en las desiguales condiciones socioeconómicas, que no son necesariamente un subproducto o un daño colateral de la crisis económica, sino que son el resultado de la aplicación sistemática de una política económica que ha ido disminuyendo el gasto social. En el caso de Chile, a la política restrictiva de gasto social que impone la concepción subsidiaria del Estado se añade un proceso de modernización económica que no es más que un mero proceso de crecimiento rentista, incapaz de ser base material para el crecimiento sostenible a largo plazo (Palma, 2010).

Un mayor deterioro material de las clases media y baja o un restringido acceso a mejores condiciones de vida responden a una intencionada transferencia regresiva de los ingresos desde los más pobres a los más ricos. La gran recesión del 2008 y las políticas de austeridad adoptadas como "solución" por los grupos de poder son las que acabaron provocando la llamada "crisis de régimen". Se trata de la pérdida de hegemonía de los sectores dominantes, expresado tanto en el quiebre de los consensos que articulaban la vida en común, como en una merma en las condiciones de vida $\mathrm{y}$, sobre todo, en la precarización de las llamadas clases medias, que actúan como sostén fundamental del sistema.

Esta convergencia brindó un campo de sutura (Laclau, 2005) en el que los más diversos problemas de la sociedad tienen un origen/adversario común: la élite gobernante o la denominada "casta" . Es lo que se puso de manifiesto en la concentración del 15M, "quienes estamos aquí somos, a buen seguro, personas muy distintas. Llevamos en la cabeza proyectos e ideales diferentes. Han conseguido, sin embargo, que nos pongamos de acuerdo en un puñado de ideas básicas", Taibo (2011).

Por este motivo, para dos de los líderes iniciales de Podemos en España, Pablo Iglesias e Iñigo Errejón, el 15M inauguró una nueva gramática política y selló las bases de construcción para un nuevo sentido común de época, una rearticulación de los consensos sociales para organizar la vida en común (Iglesias, 2015; Errejón, 2015). Una voluntad de reconstrucción de los consensos rotos por las élites que se expresa en los propios estatutos iniciales de Podemos. En dicho documento queda de manifiesto la voluntad de esta entidad de constituirse en una herramienta de los sectores populares para profundizar y avanzar en una sociedad más justa e igualitaria (Podemos, 2014). A partir de los primeros estatutos de Podemos, se abrió el terreno para la creación de un partido político que apuntó al establecimiento de una democracia radical y participativa. Una organización

Este fue el "significante vacío" que utilizó Podemos para referirse a la imbricación de intereses entre poder político y poder empresarial. 
política capaz de disputar la legitimidad establecida por el bipartidismo sobre nociones tales como democracia, Estado, nación o soberanía (Domínguez y Giménez, 2014).

En el caso de Chile, el movimiento estudiantil del año 2011 no se caracterizó por la aparente espontaneidad del $15 \mathrm{M}$ ni estuvo profusamente marcado por los efectos de la gran recesión del 2008. Sus principales demandas no apuntaron en primera instancia a una redistribución general, sino que se enmarcaron en el ámbito educativo. No obstante, vincular las inequidades del sistema educativo con una política estructural de desigualdad llevó a ampliar esta perspectiva. Esto quedó de manifiesto en uno de sus principales objetivos: "Crear un nuevo sistema nacional de educación, como un derecho social universal en todos sus niveles [...] orientado a la erradicación de las desigualdades de clase, género y etnia, a la construcción de ciudadanía y la generación de un desarrollo integral y sostenible para el país" ( $E l$ Mostrador, 2012).

Las políticas económicas que incrementaron la desigualdad socioeconómica abrieron "un espacio de significado" para impugnar el consenso racional y subvertir sus reglas y significantes. A su vez, esta impugnación es la que permite que sean otros actores los que representen las demandas de una parte de la ciudadanía que no está dispuesta a mantenerse constreñida al marco de disputa fijado por la hegemonía impugnada.

\subsection{Ruptura del consentimiento político-cultural}

Desde hace décadas, tanto Chile como España mantienen indicadores que demuestran una generalizada actitud de desinterés y desafección política. Estos indicadores, por lo demás, responden a una cultura de escasa confianza interpersonal y en las instituciones políticas (véanse Latinobarómetro, 2013; World Values Survey, 2014; Rivera-Vargas, 2016). No obstante, esta carencia de capital social no ha cuestionado la legitimidad de la democracia. De acuerdo a los estudios del CIS (2013), el 75\% de la población española prefiere la democracia a otro régimen político ${ }^{7}$. En Chile, en tanto, el $63 \%$ declara preferir la democracia (Latinobarómetro, 2013) ${ }^{8}$.

A contrario sensu, el escaso compromiso de la ciudadanía con lo político no es impedimento para el creciente apoyo a los valores de autoexpresión o postmateriales - igualdad de derechos, ecologismo, tolerancia- en ambos países (World Values Survey, 2014). Incluso, esto sucede a pesar de que los valores materiales no están completamente satisfechos, como propone una de las premisas básicas de la teoría del cambio cultural de Inglehart (1977; 2001).

En este escenario, la estructura de oportunidades políticas y los contextos de interacción y movilización de la contienda (Oñate, 2013) cobraron fuerza debido al descendente apoyo que concitaba el funcionamiento del sistema político ya antes de la crisis económica (Pérez-Nievas et al., 2013). De este modo, fueron adquiriendo mayor adhesión ciudadana las acciones extrainstitucionales - protestas, acampadas en plazas, boicots económicos, stop desahucios, recogidas

\footnotetext{
Este es un porcentaje inferior al 88,5\% registrado previo a la crisis económica (CIS, 2008).

8 Es necesario mencionar que esta legitimidad ha ido disminuyendo. De acuerdo al Latinobarómetro de 2018, este porcentaje se reduce al 57,6\% (véase en http://www.latinobarometro.org/latOnline.jsp)
} 
de firmas-, por lo que se radicalizó la participación democrática. Al igual que en un momento de coyuntura crítica, hubo una repolitización de la sociedad.

El caso del 15M resultó paradigmático en este punto. Su legitimación solo puede entenderse como respuesta a y expresión social de la crisis de régimen (Castells, 2012). Prueba de ello es que el $80 \%$ de los españoles apoyaron sus propuestas, dirigidas tanto a aspectos generales del sistema político como a la dinámica económica y social del país durante la crisis. Una de sus principales demandas fue la implantación de una democracia más participativa y alejada del bipartidismo, encarnado por el Partido Socialista Obrero Español (PSOE) y el Partido Popular (PP), y del dominio de bancos y corporaciones, además de una auténtica división de poderes y otras medidas cuya intención era mejorar el sistema democrático (Castells, 2012). Todo ello puso en tela de juicio el pacto o consenso transicional sobre el cual se edificó el régimen político de los últimos cuarenta años.

Las movilizaciones en Chile del año 2011 tuvieron impacto en la nueva configuración política del país. A juicio de Garretón (2016), pueden ser equiparables a las de Mayo del 68 francés o a las de Chiapas en 1994. En su momento, ambas expresaron una ruptura entre la política clásica y la sociedad civil. Pero estas movilizaciones también parecen cuestionar el consenso que dio origen a la dinámica política, económica y social de la postdictadura. Una dinámica que se caracterizó por los graves problemas de deuda social, una inflación no controlada por el Banco Central chileno y una exacerbada apertura económica (Morales-Olivares, 2015).

En este contexto, Podemos en España y Revolución Democrática en Chile asumieron como eje de su discurso la crítica al desempeño de las élites políticas y al relato hegemónico consagrado en las transiciones a la democracia en cada país. No cuestionan el valor de la democracia, sino el cierre excluyente del sistema político que limita la expresión a los antagonismos de intereses, valores e ideológicos (Alvarado-Espina, 2018). Parten de la premisa de que es en el terreno democrático donde se puede visibilizar una alternativa a lo que Mouffe (2012) denomina "el consenso racional". Y en ello, este discurso es central para dar forma a la realidad social (Franzé, 2017).

Para comprender la ruptura político-cultural que otorgó sentido hegemónico a la estrategia de Podemos, hay que remitirse a la reacción política de la Unión Europea ante los efectos sociales de la gran recesión de 2008, que fue precarizar la vida de las clases media y baja en la Europa meridional. Con ello, la crisis no solo afectó el modesto estado de bienestar europeo del siglo XXI, sino también la satisfacción con la democracia, especialmente en los países de la Europa meridional.

Esta ruptura acabó por instaurarse tras el vaciamiento ideológico de los viejos partidos socialdemócratas. Hay que recordar que tras la Segunda Guerra Mundial, la Europa del corto siglo XX (Hobsbawm, 2007) tuvo como principal desafío conciliar la redistribución de la riqueza en sociedades capitalistas bajo el ideario socialdemócrata y la teoría económica de Keynes. Este cambio de rumbo político consagró el estado de bienestar, el cual fortaleció la alianza entre las clases media y baja (Esping-Andersen, 1985; 1993). Pero con la crisis del petróleo de 1973, el modelo político-económico socialdemócrata comenzó poco a poco a resquebrajarse. Su caída se agudizó en la década de los ochenta. Y con el fin de la Guerra Fría acabó cediendo la hegemonía al relato ideológico neoliberal, con el que los sectores dominantes de la globalización consolidaron la desregulación del 
sector financiero y aceleraron la integración económica. En Europa, todo ello condujo al desmantelamiento de los estados de bienestar y la cesión de la soberanía monetaria a una entidad sin controles democráticos, tal como lo dispuso el Tratado de Maastricht al constituir la UE en 1994.

En este contexto, en los últimos quince años surgieron diversos procesos y movilizaciones sociales en España y el resto de Europa, donde el denominador común fue la voluntad de recuperar la soberanía nacional, la promoción de procesos de memoria histórica y la necesidad de limitar el poder de las finanzas (Fominaya y Cox, 2013). Todo esto condujo a una crisis de régimen que se develó en cuatro facetas. En primer lugar, la ruptura de los principales consensos que permitieron por décadas articular la vida en común. En segundo lugar, la emergencia de contradicciones y fracturas en el bloque dominante, especialmente debidas a los casos de corrupción que se han conocido en los últimos años (Villorria y Jiménez, 2012). En tercer lugar, las políticas de austeridad, que conllevan un ensañamiento con los sectores populares a través de recortes, desempleo, pérdida de poder adquisitivo, disminución de libertades civiles y políticas (Navarro, 2012). Finalmente, en un cambio del sistema de partidos justo después del estallido del $15 \mathrm{M}$, en $2011^{9}$. Esto supuso, por primera vez en décadas, una ruptura de la lógica de vasos comunicantes que mantenía vigente al bipartidismo (Monedero, 2012).

Esta crisis brindó una oportunidad para cambiar el sentido de época. Tras las elecciones del 26 de junio de 2016, Pablo Iglesias señaló que era necesario consolidarse como el "motor de un espacio político de varias identidades" y construir un "bloque histórico con movimientos populares" (Público, 2016). Esta declaración está en línea con aquello de que todo nuevo grupo social homogéneo debe definir su propia filosofía (o ideología) y combatir el sentido común predominante (Portelli, 1977).

Por otra parte, existe un diagnóstico compartido sobre el agotamiento del marco político-cultural construido durante las respectivas transiciones políticas. Podemos redefinió el periodo histórico que surge de la Transición como Régimen del 78', mientras que Revolución Democrática ${ }^{10}$ desdeña los acuerdos fundantes del consenso transicional que se consagran en la década de los noventa del siglo pasado, a los que define como elitistas. En palabras de Mayol (2016), el clivaje derecha versus concertación operó como un pacto elitario que logró salvaguardar la herencia dictatorial en materia económica e institucional.

Es así como, en ambos casos, el proceso transicional se identifica con una autotransformación de la dictadura —utilizó su propia legalidad para llevar adelante un cambio político legitimado por el concurso de la oposición democrática de la época- y un buen arreglo para las élites económicas (Alvarado-Espina, 2017). Esta diagnosis fue fuente para un nuevo relato; un nuevo sentido común que impugna el consenso que originó el pacto vigente entre sociedad civil y sociedad política.

En el caso de España, la autoatribuida dinámica consensual de su Transición se construyó en un escenario con dos importantes características. En primer lugar, se trató de un proceso político marcado tanto por la crisis del petróleo (y sus

$9 \quad$ En las elecciones generales de 2011, el Partido Popular ganó con mayoría absoluta, mientras que el PSOE perdió el $40 \%$ de su apoyo electoral.

10 Este partido nace como un movimiento político el 7 de enero de 2012. Sus principales fundadores son líderes de la movilización estudiantil de 2011, entre los que destaca el diputado Giorgio Jackson. Se define como un movimiento de izquierda política que busca la construcción de un socialismo democrático y la democracia participativa. 
consecuencias a nivel mundial) y un fuerte componente de movilización política contra la dictadura. En segundo lugar, se trató de un proceso conducido por las élites políticas y económicas configuradas durante el franquismo. Por este motivo, para Errejón la Transición española fue "una maniobra de restauración ampliada de la hegemonía, que oxigena el pluralismo e incorpora, en forma subordinada, a otros grupos sociales al bloque de poder [...] y fragua un relato y una intelectualidad que solidifican el nuevo orden" (Errejón y Mouffe, 2015: 33). A partir de esta perspectiva, Podemos llegó a plantear la transformación del régimen político consolidado en 1982 (Soto, 2003).

Por su parte, Revolución Democrática fue más mesurada en sus inicios en la oposición al régimen gobernante de la Concertación, participando incluso de pactos por omisión en algunas decisiones. No obstante, la opinión generalizada se asemeja a la crítica de acomodación que caracterizó la transición chilena, donde existió una inconsistencia sostenida entre el discurso socialdemócrata y la praxis política neoliberal (Morales-Olivares, 2015).

En ambos casos, se construye un relato político alternativo y potencialmente emancipador que apela a las clases populares, por un lado, pero que se extiende a las clases medias, a los desfavorecidos de la crisis económica en España y a la mayoría olvidada del modelo neoliberal en Chile. A través de este relato, las nuevas formaciones políticas intentan suturar los diferentes intereses, valores y demandas que se encuentran fuera del sistema político, con el objeto de darles una voz política de ruptura hegemónica al interior del sistema.

\section{Factores institucionales y no institucionales de consolidación}

\subsection{Un sistema político desgastado, aunque con fuertes barreras de entrada}

Para calibrar mejor la perspectiva de análisis, hay que destacar algunas diferencias político-institucionales que existen entre ambas democracias. En primer lugar, se encuentra la forma de gobierno. España se organiza políticamente en torno a un régimen monárquico parlamentario, mientras que Chile lo hace en torno a una república presidencial. En el primero, los partidos políticos cumplen un rol primordial en la designación final del jefe de Gobierno, mientras que en el segundo ese rol se limita a la nominación de los candidatos que compiten por la jefatura de Estado. Por lo tanto, el grado de incidencia de los partidos en las políticas del Gobierno es más preeminente en el país europeo que en el sudamericano.

En segundo lugar, en España la competencia electoral se sustenta en los partidos con una alta preponderancia de los dirigentes nacionales. Son ellos los que confeccionan las listas que, por lo además, son cerradas. En Chile, en tanto, la competencia electoral está determinada por candidaturas individuales al interior de los partidos. Este factor es importante para entender el nivel de incidencia que uno u otro partido puede alcanzar en cada país.

Por último, hay un tercer elemento que distingue la realidad política de ambos países: la Unión Europea. La pertenencia de España a este espacio de gobernanza supranacional implica, entre otras cosas, elegir miembros a su Parlamento. Este hecho permite a partidos en formación, o de escasa presencia institucional en sus 
respectivos países, probar suerte electoral. Es lo que Podemos hizo en mayo de 2014 con gran éxito al superar todas las expectativas demoscópicas. La obtención de cinco eurodiputados y cerca de un millón y medio de votos le convirtió en la cuarta fuerza política más votada en España, al tiempo que, por primera vez en este tipo de elecciones, los dos partidos mayoritarios sumandos - PP y PSOE - no alcanzaron el $50 \%$ de los sufragios.

Considerando las diferencias de contexto institucional en las que se desenvuelven los dos nuevos partidos, se compara la inserción y consolidación política de cada uno a partir de la principal dimensión procesal del régimen liberal y democrático que rige en ambos países: la selección de cargos políticos vía sufragio universal. Para hacerlo, se comparan los resultados electorales obtenidos por sus respectivos pactos electorales, en las elecciones generales al Congreso de los Diputados realizadas en España en 2011, 2015, 2016 y 2019, y las elecciones parlamentarias (diputados) en Chile de 2009, 2013 y $2017^{11}$.

En términos metodológicos, para esta comparación se utiliza el porcentaje de votos obtenidos por los partidos que consiguieron escaños en cada elección. Esto permite observar el nivel de impacto que tienen Podemos y Revolución Democrática, tanto en la hegemonía de la representación política que tenían los partidos tradicionales como en el realineamiento de preferencias de los electores. Esto también ayuda a conocer la evolución de los apoyos que reciben en cada elección. Aunque esto se puede observar mucho mejor en el caso de Podemos, ya que RD compitió como partido por primera vez en 2017.

Al comparar las elecciones realizadas en España durante el periodo 2011-2019 en el gráfico 1 destacan dos hechos. El primero, se produjo un realineamiento en las preferencias tanto en el electorado de izquierdas como en el de derechas. Esto supuso, por ejemplo, un bolsón de votos importante para un partido nuevo como Podemos, pero también para otro preexistente, aunque periférico, como Ciudadanos ${ }^{12}$. Segundo, se produjo la fatiga de varias formaciones políticas menores que acabaron cediendo o asimilando su representación política a los llamados partidos emergentes. Estos fueron los casos de UPyD, GBAI, Compromís e IU. Este último caso resulta particularmente relevante, considerando que una parte no menor de los fundadores de Podemos, antes del 15M, militó activamente en esta entidad (Monedero, 2016).

Ambos hechos reflejan un movimiento centrípeto del sistema político español que paradójicamente puso fin al bipartidismo.

En lo que se refiere a las barreras de entrada, la pronunciada caída en el apoyo electoral a los dos grandes partidos (PP y PSOE) condujo a un reordenamiento en la representación política institucional. Así, y a pesar de la notable desproporcionalidad que exhibe el sistema electoral español en favor de las dos grandes formaciones políticas de derecha y centroizquierda, fue posible el ingreso de nuevos partidos al Congreso que condicionó la formación de gobierno. Eso sí, este fenómeno tuvo una contracción en las elecciones generales de abril de 2019,

11 En el porcentaje de votación global de Podemos, están incluidas sus marcas autonómicas en Catalunya (En Comú Podem), Galicia (En Marea) y País Valencià (coalición Podem-Compromís) en 2015. A estas se suma Izquierda Unida en 2016, partido que concurrió a las elecciones en coalición con Podemos. Para el caso de Revolución Democrática, se considera la votación obtenida de forma individual.

12 Este partido, fundado en 2006, nunca había competido a escala estatal. Hasta 2015 su espacio político se circunscribía exclusivamente a Cataluña. 
cuando se produjo una caída en la votación de Unidas Podemos que pareció favorecer la subida del PSOE.

Gráfico 1. Preferencias electorales en España, 2011-2019



Fuente: elaboración propia a partir de los datos del Ministerio de Interior, obtenidos en http://www.infoelectoral.mir.es/infoelectoral/min/

Para comprender el impacto de la llegada de RD a las instituciones de representación política, hay que considerar la relación que se da entre la irrupción de los liderazgos políticos universitarios en 2011 y el cambio del sistema electoral en 2015.

El sistema electoral binominal que regía en Chile hasta las elecciones generales de 2013 obligaba a los partidos a agruparse en pactos preelectorales. Esto no promovía la competencia fuera de los pactos y restringía la representación política a dos grandes mayorías (véase Alvarado-Espina, 2015; Huneeus et al., 2015). Esta fue una barrera de entrada insuperable para nuevos partidos o partidos minoritarios. De hecho, el Partido Comunista obtuvo sus primeros diputados en 2009 al incorporarse a la coalición de partidos de la Nueva Mayoría. Lo mismo sucedió con los dirigentes estudiantiles Jackson, Vallejo y Boric, quienes obtuvieron un escaño en el Congreso al concurrir a la elección parlamentaria de 2013 dentro de ese mismo pacto.

La incorporación de estos nuevos parlamentarios, que provenían del movimiento estudiantil, actúo como aliciente en el Gobierno de Bachelet (2014-2018) para reformar el sistema electoral, al aumentar la magnitud de los distritos y ampliar el número de Diputados ${ }^{13}$. El nuevo sistema electoral permitió que sectores políticos que no participaban de las dos coaliciones mayoritarias accedieran al Congreso.

\footnotetext{
13 La Ley 20.840 pone fin al sistema electoral binominal creando un sistema proporcional inclusivo.
} 
Gráfico 2. Preferencias electorales en Chile, 2009-2017

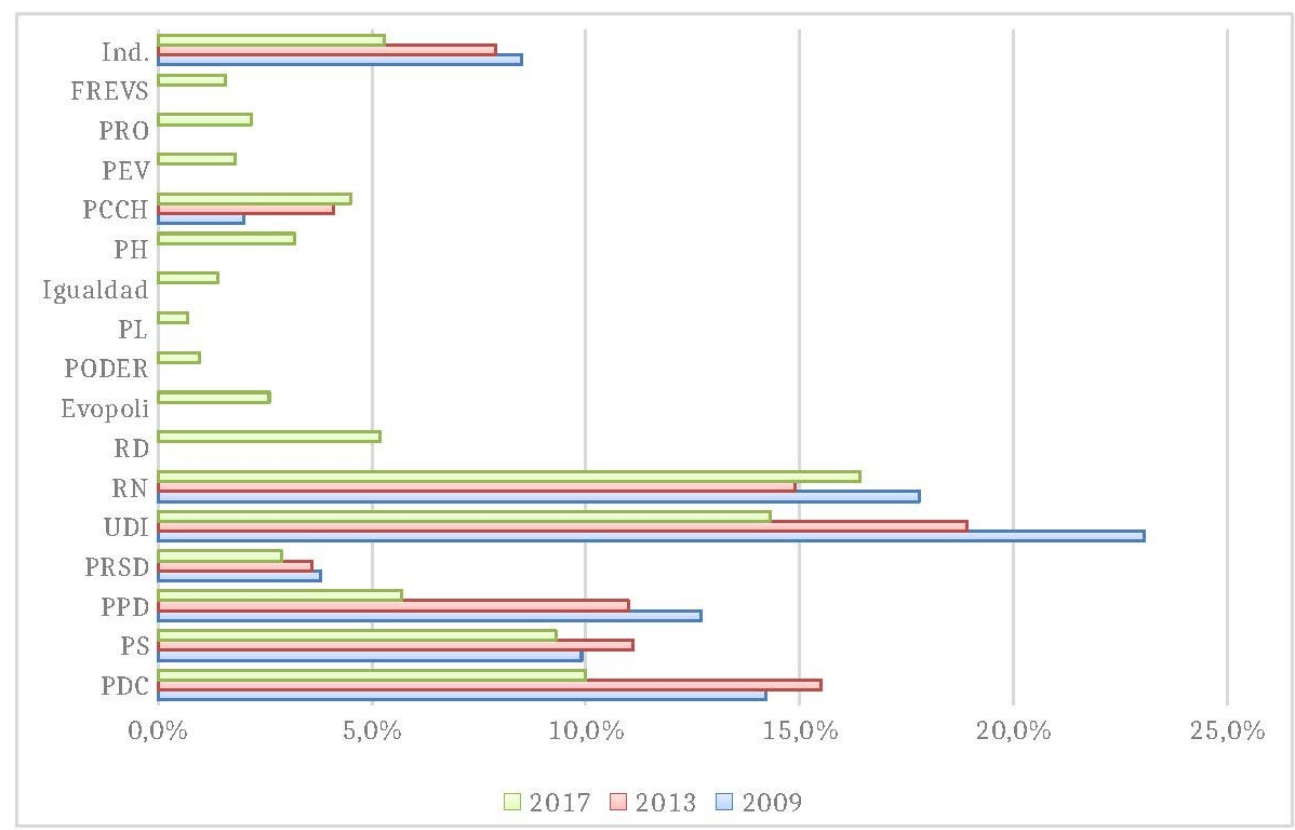

Fuente: elaboración propia a partir de los datos del Servicio Electoral, obtenidos en $\mathrm{http}: / / \mathrm{pv}$.servelelecciones.cl/ y https://historico.servel.cl/

Como se puede observar en el gráfico 2 a irrupción de RD y del Frente Amplio en el ámbito institucional provocó una merma en el apoyo de los electores de centro-izquierda a los partidos tradicionales que se sitúan en ese espectro ideológico. Así, el 5,2\% y los diputados obtenidos por RD en 2017 parecen provenir de electores que en el pasado votaron por partidos como el PS, PPD, PRSD o PDC. Todos estos partidos muestran una significativa caída si se compara la elección de diputados de 2017 con la de 2013. Este movimiento de electores tuvo efectos en la representación política, lo que también se debe al cambio del anterior sistema electoral por otro de carácter proporcional inclusivo.

En síntesis, en los gráficos 1 y 2 se constata que tras la irrupción de nuevos actores sociales que condicionaron el debate en la esfera pública, el sistema político comenzó a abrirse a nuevas opciones políticas. En ese proceso, los partidos tradicionales fueron perdiendo injerencia en la agenda pública, al tiempo que sus líderes comenzaron a ser desplazados por un tipo de liderazgo más transversal proveniente de los movimientos sociales y las organizaciones de la sociedad civil.

Estos cambios de la dinámica política no solo favorecieron la emergencia de nuevos partidos políticos, también modificaron las preferencias de los electores. Esto último es lo que permitió a Podemos y Revolución Democrática alcanzar una importante representación política. Quizás la mayor diferencia estriba en que Podemos, a diferencia de Revolución Democrática, ya estaba relativamente 
consolidado un año antes de presentarse a sus primeras elecciones generales, debido a su desempeño en las elecciones europeas de 2014.

Este desplazamiento en las preferencias políticas de una parte importante del electorado politizado, e incluso despolitizado en España, vino a demostrar la conexión que existe entre intereses y valores no representados por los partidos tradicionales y la consolidación del partido-movimiento. Un efecto que se agudizó con el deterioro de las condiciones materiales en la España poscrisis y la desigualdad estructural en el acceso a los bienes sociales que consolidó el Gobierno de Sebastián Piñera en Chile. Ahora bien, este proceso solo se correlaciona con una mayor movilización electoral en España, no en Chile.

Finalmente, el paso desde un formato no convencional de partido (partido-movimiento) a uno convencional tuvo en el resultado electoral una de sus causas. En el caso de Podemos, fueron sus propios dirigentes los que anunciaron que el partido adoptaría un modelo más convencional tras las elecciones del 26J (Franzé, 2017).

\subsection{Acciones políticas disruptivas que definen la constitución del partido}

De acuerdo al enfoque de la democracia radical, es en el espacio de lo político donde se definen los antagonismos, y la búsqueda de las hegemonías se cristaliza en los movimientos que impugnan el statu quo (Mouffe, 2012; Errejón y Mouffe, 2015). Por tanto, y concordando con Tarrow (1997), los movimientos sociales pueden representar una acción colectiva de resistencia, desafío y alternativa al orden social predominante. Incluso pueden dar forma a un proyecto político alternativo en un momento de ruptura del consentimiento otorgado al régimen político y a sus élites. Eso sí, siempre en la medida en que cristalicen en un nuevo discurso de realidad.

El principal elemento que comparten Podemos y Revolución Democrática es que a ambas formaciones políticas les preceden potentes movimientos sociales que destacaron por impugnar el poder de la lógica discursiva neoliberal. Estas nuevas formas de movilización social, asociadas al cambio cultural de la sociedad posindustrial (Inglehart, 1977; 2001), son las que facilitaron la emergencia de nuevos actores y redes formales e informales de movilización que perjudicaron a las viejas élites y los vehículos tradicionales de participación (Oñate, 2013). En definitiva, en ambos casos la movilización social fue el semillero de un producto político permanente.

Podemos se constituyó a partir de dos vertientes: 1) las complicidades que generó el 15M entre militantes de partidos minoritarios de izquierdas y líderes de organizaciones sociales; y 2) el proceso autoconvocado para la creación de círculos en todo el país, fundado en la mera voluntad de participar de cada individuo. A primera vista, Podemos encajaría en la clasificación de partido-movimiento, básicamente porque su éxito inicial no puede concebirse ni entenderse sin la existencia previa del movimiento $15 \mathrm{M}$, aunque no hay que dejar de lado el soporte de otras pequeñas organizaciones políticas surgidas antes y después de la primavera de 2011 (Meyenberg, 2017).

En su evolución política hacia un partido convencional, Podemos comenzó por centralizar la toma de decisiones en sus órganos de dirección. Como se desprende de su documento organizativo "Mandar obedeciendo", aprobado en la Asamblea 
Ciudadana de Vistalegre 2, la versión más horizontal e instrumental plasmada en su fundación pierde centralidad. Aun así, se mantiene como principio rector la igualdad de todos sus afiliados en la toma de decisiones y una preponderancia de la Asamblea Ciudadana (Podemos, 2017).

Por su parte, Revolución Democrática emerge en 2012 como un movimiento político articulado en torno a un grupúsculo de dirigentes estudiantiles, organizaciones sociales e intelectuales ubicados al margen del poder institucional. Durante este proceso se asoció a otro grupo político de origen estudiantil y de izquierdas, el Movimiento Autonomista. Entre ambos dieron forma a una nueva coalición política denominada Frente Amplio. La evolución de esta alianza se convirtió en el puntapié inicial de la convergencia de otras fuerzas políticas, como los partidos Comunes, Humanista, Igualdad y Liberal.

$\mathrm{Al}$ alero del movimiento universitario, Revolución Democrática se perfiló como un movimiento político ad hoc a los planteamientos surgidos de las movilizaciones estudiantiles de 2011, y que tenía como primer objetivo político conseguir que su máximo dirigente fuera elegido diputado ${ }^{14}$ (Concha, 2013). En los terrenos programático y electoral, se presentó como una alternativa a los tradicionales partidos de centro-izquierda agrupados en la coalición Nueva Mayoría.

Ahora bien, si se compara con Podemos, Revolución Democrática no contó con una organización territorial en todo el país, ni con una implantación similar a la que ese partido consiguió en un año en España. Solo se presentaron algunos casos puntuales de organización de base o barriales.

$\mathrm{RD}$ se autodefine como un espacio capaz de aunar movimiento social y política institucional, con la pretensión de superar el orden neoliberal impuesto en la sociedad chilena a través de la profundización democrática. Su organización ha sufrido cambios desde su fundación, y hoy en día es un partido con un proceso de toma de decisiones más tradicional o clásico. A partir de su constitución como partido en 2016, su estructura organizacional se edificó en cuatro órganos colegiados, cuyos procesos de toma de decisión no están sometidos al escrutinio de sus militantes de forma permanente. A diferencia de Podemos, no se contempla una instancia política decisional en la que participen todos sus militantes (véase, Revolución Democrática, 2017).

En ambos casos sucede que la interlocución discursiva es directamente hacia la gente o ciudadanía como contraparte de la élite o "casta" política. Esta es una estrategia que resulta útil para solventar momentáneamente dos problemas que pueden dificultar una acción política colectiva contrahegemónica, la heterogeneidad de intereses y las singularidades valóricas. A través de este discurso se promueve el sentido común con el que se construye el propio terreno de disputa - el pueblo - en los términos planteados por Gramsci (1975) y rescatados por Errejón y Mouffe (2015).

En función a la organización y al alcance del discurso, estos actores políticos introdujeron algunos cambios disruptivos en su primera fase de consolidación, como el realineamiento de los votantes y una recomposición general en la distribución del poder institucional. También, un modelo de financiación de

14 Giorgo Jackson, expresidente de la Federación de Estudiantes de la Universidad Católica y uno de los fundadores del movimiento Revolución Democrática. 
actividades políticas al margen del sistema bancario y la utilización del espacio público como punto de reunión y organización política.

En España, la irrupción de Podemos y sus confluencias ${ }^{15}$ provocó tres importantes cambios en el sistema político español. El primero, un realineamiento de los votantes de izquierdas en dos bloques con similar apoyo electoral. El segundo, acabó con la lógica bipartidista del sistema político como consecuencia de lo primero. Y el tercero, impulsó un nuevo sector de izquierda sumando votantes de la izquierda más tradicional, especialmente de Izquierda Unida.

En Chile, Revolución Democrática y su coalición política - Frente Ampliotambién demostraron su capacidad para provocar cambios disruptivos. Uno de ellos fue aminorar considerablemente la exclusión parlamentaria de los partidos políticos que no participaban de los dos grandes pactos electorales. Otro fue la introducción de demandas sociales que no contaban con representación política institucional, por ejemplo la petición de rebaja de la dieta parlamentaria, que supera en 11 veces el ingreso per cápita del país ${ }^{16}$.

Pero existen algunas diferencias en la organización primigenia de Podemos y Revolución Democrática como partidos-movimiento. Aunque en ambas experiencias se da la convergencia de partidos y grupos políticos minoritarios de izquierdas, su consolidación e impacto no son los mismos. Podemos está presente en casi todo el territorio español, como fruto de un proceso autoconvocado de ciudadanos y organizaciones sociales. Esto le permitió asentarse, en las elecciones municipales, autonómicas y generales como opción política a los partidos tradicionales que han gobernado España desde 1982. Con Revolución Democrática sucede algo similar. Esta incipiente organización, dentro del Frente Amplio, también logró representar una alternativa al oligopolio político que se consolidó en Chile en 1990, aunque con una menor intensidad que Podemos.

En términos generales, Podemos y Revolución Democrática presentan semejanzas en su discurso contrahegemónico basado en la dicotomía pueblo/élites, donde ambos proyectos políticos apelaron a la construcción de un nuevo sentido común — los de arriba frente a los de abajo - ; y, por otra parte, se identificación con los valores postmateriales: igualdad de género, derechos de las minorías, protección del medio ambiente, tolerancia y no discriminación.

\section{Conclusiones}

En este trabajo, en primer lugar se han explorado las razones que explican la emergencia y consolidación de dos nuevos partidos políticos emergentes: Podemos en España y Revolución Democrática en Chile. En este marco, también, se han analizado sus potencialidades en tanto partidos-movimiento, considerando las condiciones socioeconómicas, políticas, culturales e institucionales que pudieron incidir en su configuración.

La perspectiva de la democracia radical y del pluralismo agonístico ha sido el fundamento teórico del análisis. En tal sentido, se toma en cuenta el

En Comú Podem en Cataluña, Podem en País Valencià y En Marea en Galicia.

16 Esta ha sido una de las demandas más sentidas del movimiento social que emerge tras la explosión social del 18-O de 2019. De hecho, el 27 de noviembre se ha aprobado por unanimidad la rebaja de un 50\% de esta dieta en la Cámara de Diputados. 
autorreconocimiento de la dimensión conflictiva que define al régimen liberal democrático como un aspecto dinamizador de la deliberación política y la resignificación de la sociedad (Mouffe, 2012). Se propugna una relación de antagonismos políticos y sociales que pudieron dar origen a dos nuevos partidos políticos que parecen encajar en la tipología de partido-movimiento de Kitschelt (2006).

A partir de este marco interpretativo comparado, se configuran dos dimensiones de análisis para explicar la emergencia y consolidación de un partido-movimiento: 1) la profundización estructural de los antagonismos y 2) la explosión de un proceso de deconstrucción de la hegemonía ideológica dominante. Del análisis comparado, se obtienen algunas respuestas a los tres interrogantes planteados.

En relación a la primera pregunta, que buscaba conocer la existencia de factores estructurales determinantes para que los movimientos sociales del año 2011 dieran paso a la formación de nuevos partidos políticos, se puede responder que sí los hubo. A pesar del contraste en las trayectorias socioeconómicas durante el último lustro, el aumento de la desigualdad social en España, así como su perpetuación en Chile, genera un espacio de intereses bastante propicio para la impugnación del régimen político. En ese espacio, los movimientos sociales de 2011 fueron la cerusa que visibilizó la frontera de antagonismos trazada entre los que disfrutan de los beneficios y privilegios del sistema económico y los que pagan los costes de sus diferentes crisis. Esa visión más nítida de la frontera social fue lo que resquebrajó el consentimiento mayoritario que la ciudadanía otorgaba al consenso ideológico de las élites políticas. De este modo, surge la oportunidad para propagar un relato contrahegemónico o nuevo sentido común que buscó crear pueblo.

En relación a la segunda pregunta, que intenta establecer si ambos partidos encajan en las características o dimensiones del partido-movimiento de Kitschelt (2006), podemos decir que así es. Su origen "desde abajo", lo que en parte contradice la afirmación de Mair (2015) respecto a la retirada de los ciudadanos de los asuntos públicos y la política, es la característica básica de este tipo de organizaciones políticas. Una dirección basada en el liderazgo carismático, una toma de decisiones más horizontal, procesos de conformación programática más amplios y una selección de candidatos abierta a todos los inscritos en el partido lo confirman. Sin embargo, esto solo sucedió en una primera etapa. Tras su consolidación política y electoral, ambos partidos iniciaron un proceso de reconfiguración estratégica que los ha ido convirtiendo paulatinamente en organizaciones políticas más permanentes y convencionales.

Por último, en relación a la tercera pregunta, que buscaba determinar si estos nuevos actores políticos se han consolidado como una alternativa a los partidos tradicionales, se puede decir que al no responder a la lógica tradicional de los actuales partidos "cartel" (Katz y Mair, 2004), además de la disrupción que han provocado en el sistema político, los convierte en una alternativa plausible dentro de los cauces democráticos. Como se ha constatado en el análisis, existen dos hechos que lo demuestran. El primero, han conseguido representar a una parte importante de la sociedad gracias a su forma de organización - desde abajo y sin apoyo del sistema financiero- y a un discurso que estructuró el malestar en una opción política. El segundo, su concurso electoral provocó un realineamiento de los 
electores de centro-izquierda que les encumbró como eje principal de una tercera fuerza política en liza por el Gobierno.

Si bien la construcción de partidos que disputen el relato hegemónico, respetando los valores liberales del régimen democrático, es compleja e improbable, parece altamente probable que ambos conglomerados tengan la posibilidad de consolidar los espacios que han ganado para la divulgación política, tanto a nivel parlamentario como local.

La práctica y convicción de estas transformaciones es uno de los ejercicios sustantivos del hacer político, con unos hitos políticos -Podemos y sus confluencias son la tercera fuerza parlamentaria y Revolución Democrática es parte de una coalición política que ya es la tercera fuerza parlamentaria- que no estaban en la agenda de los analistas. No se pueden dejar de considerar las probabilidades de consolidación que brinda el contexto socioeconómico y cultural.

En síntesis, génesis y constitución de Podemos y Revolución Democrática parecen responder al concepto partido-movimiento, aunque sin seguir necesariamente el mismo patrón. Y a pesar de que sus proyectos se erigen al margen del sentido común instaurado en cada transición, su grado de institucionalización confirmará si mantienen ese signo de partido alternativo e impugnación al régimen político o se convierten en una alternativa más dentro del mismo régimen.

A simple vista, parece contraintuitivo que dos países de renta media y media-alta y con escasa cultura cívica puedan dar espacio a nuevas organizaciones políticas que cuestionan el funcionamiento del régimen político y el sistema social. Sin embargo, esto sería así desde el prisma liberal, ya que este fenómeno viene a demostrar que existe una dialéctica política entre las condiciones socioeconómicas y la respuesta que otorga el régimen político ante el empeoramiento de esas condiciones. Ambos aspectos son elementos causales de la apertura de nuevas significaciones políticas y consolidación de opciones políticas de ruptura que persiguen cambiar - y cambian en la praxis - las reglas del juego en que se sostiene la relación entre política, economía y sociedad civil, por una parte, y entre estructura económica y superestructura ideológica, por otra.

De esta forma, lo que persiguen Podemos y Revolución Democrática, tal como se ha analizado, es disputar el terreno del sentido común dando espacio a nuevas significaciones radicales, que incluyen un componente de clase importante. Sin embargo, no los definen de una vez y para siempre en una única identidad, pues permiten - en tanto partidos movimientos - la contingencia y solidificación desde abajo de sus demandas y propuestas institucionales.

\section{Bibliografía}

Alvarado-Espina, E. (2015): “Exclusión, oligarquización y desafección política. Los efectos del sistema binominal en la democracia chilena", Enfoques, 13 (22), pp. 11-37.

Alvarado-Espina, E. (2017): "La calidad de la democracia en España y Chile. Un estudio a partir de la desigualdad y el elitismo político", Tesis doctoral inédita, Universidad Complutense de Madrid, Madrid.

Alvarado-Espina, E. (2018): "Una aproximación crítico-contextual al declive de la democracia en la era neoliberal", Revista Española de Ciencia Política, 47, pp. 69-91.

Castells, M. (2012): Redes de indignación y esperanza, Madrid, Alianza Editorial. 
CIS. (2008): Estudio 2778 de noviembre de 2008 (Barómetro). Disponible en: http://www.cis.es/cis/export/sites/default/-Archivos/Marginales/2760_2779/2778/ e277800.html [Consulta: 20 de enero de 2018]

CIS. (2013): Estudio 3007 de noviembre de 2013 (Latinobarómetro XIV). Disponible en: http://www.cis.es/cis/export/sites/default/-Archivos/Marginales/3000_3019/3007/ es3007mar.pdf [Consulta: 20 de enero de 2018]

Concha, L. (2013). "La hoja de ruta de Jackson y Revolución Democrática”, en La Tercera. Disponible en: https://revoluciondemocratica.cl/la-hoja-de-ruta-de-jackson-y-revolucion-democratica/ [Consulta: 7 de enero de 2018]

Domínguez, A. y L. Giménez (2014): Claro que Podemos. De La Tuerka a la esperanza del cambio en España, Barcelona, Los libros del lince.

El Mostrador (2012): "Las 'exigencias' del movimiento estudiantil al gobierno y parlamentarios para un 'nuevo Sistema Educacional'”, Disponible en: http://www.elmostrador.cl/noticias/pais/2012/06/28/las-exigencias-del-movimientoestudiantil-al-gobierno-y-parlamentarios-para-un-nuevo-sistema-educacional/ [Consulta: 12 de diciembre de 2017]

Errejón, Í. y Ch.Mouffe (2015): Construir pueblo. Hegemonía y radicalización de la democracia, Barcelona, Icaria.

Errejón, I. (2015): "PODEMOS como práctica cultural emergente frente al imaginario neoliberal: hegemonía y disidencia. Conversación con Íñigo Errejón”, IC Revista Científica de Información y Comunicación, 11, pp. 17-46.

Esping-Andersen, G. (1985): Politics against markets: The social democratic road to power, Princeton, Princeton University Press.

Fominaya, C. F. y L. Cox (2013): Understanding European movements: New social movements, global justice struggles, London, Routledge.

Franzé, J. (2017): La trayectoria del discurso de Podemos: del antagonismo al agonismo, Revista Española de Ciencia Política, 44, pp. 219-246.

Garretón, M. A. (2016): La gran ruptura: Institucionalidad politica y actores sociales en el Chile del siglo XXI, Santiago, LOM.

Gramsci, A. (1975): Cartas desde la cárcel, Madrid, Cuadernos para el diálogo.

Hobsbawm, E. (2007): Guerra y paz en el siglo XXI, Barcelona, Crítica.

Iglesias, P. (2015): “Understanding Podemos”, New Left Review, 93, pp. 7-22.

Huneeus, C., M. Lagos y A. Díaz (2015): Los dos Chiles, Santiago, Catalonia.

Inglehart, R. (1977): Silent Revolution: Changing Values and Political Styles among Westerns Publics, New Jersey, Princeton University Press.

Inglehart, R. (2001): Modernización y posmodernización: el cambio cultural, político y económico en 43 sociedades, Madrid, CIS.

Katz, R. y P. Mair (2004): "El partido cartel. La transformación de los modelos de partidos y de la democracia de partidos", Zona Abierta, 108/109, pp. 9-41.

Kitschelt, H. (2006): “Movement Parties", en R. Katz y W. Crotty, eds., Handbook of Party Politics, London, Sage, pp. 278-290.

Laclau, E. y Ch. Mouffe (1987): Hegemonía y estrategia socialista. Hacia una radicalización de la democracia, Madrid, Siglo XXI.

Laclau, E. (2005): La Razón Populista, Buenos Aires, Fondo de Cultura Económica. 
Latinobarómetro (2013): Informe 2013, Disponible en:

http://www.latinobarometro.org/latOnline.jsp

[Consulta: 10 de noviembre de 2017]

Latinobarómetro (2018): Informe 2018, Disponible en:

http://www.latinobarometro.org/latContents.jsp

[Consulta: 30 de octubre de 2019]

Leroux, S. y J. C. Morales Guzmán (2016): "Democracia y desencanto: problemas y desafíos de la reconstrucción democrática del estado. Entrevista a Luis Salazar Carrión", Andamios, 13 (30), pp. 135-153.

Lobera, J. (2015): "De movimientos a partidos: la cristalización electoral de la protesta", Revista Española de Sociología, 24, pp. 97-105.

Mair, P. (2015): Gobernando el vacio. La banalización de la democracia occidental, Madrid, Alianza.

Martín, I. (2015): "Podemos y otros modelos de partido-movimiento", Revista Española de Sociología, 24, pp. 107-114.

Merkel, W. (2014): “Is capitalism compatible with democracy?", Zeitschrift für Vergleichende Politikwissenschaft, 8 (2), pp. 109-128.

Ministerio de Interior. (s.f.): Resultados elecciones generales 2011, 2015, 2016 y 2019. Disponible en:

http://www.infoelectoral.mir.es/infoelectoral/min/

[Consulta: 10 de abril de 2018]

Monedero, J. C. (2012): “¿Posdemocracia? Frente al pesimismo de la nostalgia, el optimismo de la desobediencia", Nueva Sociedad, 240, pp. 68-86.

Monedero, J. C. (2016): "Las debilidades de la hipótesis populista y construcción de un pueblo en marcha", Madrid, Público.

Morales-Olivares, R. (2015): Inconsistencies between social-democratic discourses and neo-liberal institutional practices in Chile and South Africa: a comparative analysis of the post-authoritatarian periods", Peter Wagner ed. African, American and European trayectories of modernity, Edinburgh: Edinburgh University Press, pp. 125-148.

Mouffe, Ch. (1998): Desconstrucción y pragmatismo, Paidós, Buenos Aires.

Mouffe, Ch. (1999): El retorno de lo político: comunidad, ciudadanía, pluralismo y democracia radical, Barcelona, Paidós.

Mouffe, Ch. (2012): La Paradoja Democrática: El Peligro del Consenso en la Política Contemporánea, Barcelona, Gedisa.

Navarro, V. (2012): "El error de las políticas de austeridad, en la sanidad pública”, Gaceta Sanitaria, 26 (2), pp. 174-175.

OECD (2016): ¿Cómo va la vida en España? Better Life Iniciative. Paris: OECD. goo.gl/FXHbWJ

OECD (2017): Gasto Social en los países miembros de la OECD. Disponible en: https://data.oecd.org/socialexp/social-spending.htmy

[Consulta 14 de diciembre de 2017]

Oñate, P. (2013): "La movilización ciudadana en España en los albores del siglo XXI: una contextualización para el debate", Revista Española de Ciencia Política, 33, pp. 31-55.

Palma, J. G. (2010): "Why has productivity growth stagnated in most Latin American countries since the neo-liberal reforms?", en José Antonio Ocampo y Jaime Rose eds., The Handbook of Latin American Economics, Cambridge, University of Cambridge, pp. 568-608. 
Pérez-Nievas, S. et al. (2013): Los efectos de la crisis económica en la democracia española: legitimidad, insatisfacción y desafección, Universidad Autónoma de Madrid, Madrid.

Piketty, T. (2015): El capital en el siglo XXI, Barcelona, RBA libros.

PODEMOS (2014): Estatutos del Partido Político Podemos. Disponible en: https://participa.podemos.info/pdf/estatutos_de_podemos.pdf

[Consulta 28 de noviembre de 2018]

PODEMOS (2017): Documento Organizativo. Asamblea Ciudadana Vistalegre II. Disponible en:

https://files.podemos.info/9AM13us6iC.pdf

[Consulta: 28 de Octubre de 2019]

Portelli, H. (1977): Gramsci y el bloque histórico, Madrid, Siglo XXI.

Revolución Democrática (2017): Título III: Organización Interna del Partido. Disponible en: https://www.portaltransparencia.cl/PortalPdT/documents/ 10179/62801/2019_02_22+Extracto+Estatuto+2017.pdf/01114ec7-de57-40e2-bf51e4b5b8cebd53

[Consulta: 30 de octubre de 2019]

Rivera-Vargas, P. (2016): "Modernización reflexiva en Chile: Participación electoral y confianza de la ciudadanía en las instituciones durante la Post Dictadura. Análisis entre los años 1999-2010", Políticas Públicas, 2 (9), pp. 90-108.

Servel. (s.f.): Resultados elecciones de diputados 2009 y 2013. Disponible en: https://historico.servel.cl/ [Consulta: 15 de marzo de 2018]

Servel. (s.f.): Resultados elección de diputados 2017. Disponible en: http://pv.servelelecciones.cl/ [Consulta: 15 de marzo de 2018]

Taibo, C. (2011): “Indignación e indignados del 15M”, Retóricas. Disponible en: http://www.retoricas.com/2011/06/indignados-del-15-m-carlos-taibo.html

Tarrow, S. (1997): El poder en movimiento. Los movimientos sociales, la acción colectiva y la política, Madrid, Alianza Universidad.

Villoria, M. y F. Jiménez (2012): “La corrupción en España (2004-2010): datos, percepción y efectos”, Revista Española de Investigaciones Sociológicas, 138(1), pp. 109-134. 